\title{
Measurement and simulation of laser power noise in GEO 600
}

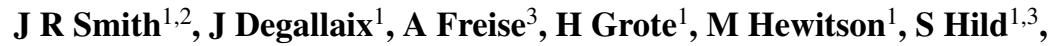 \\ H Lück ${ }^{1}$, K A Strain ${ }^{4}$ and B Willke ${ }^{1}$ \\ ${ }^{1}$ Max-Planck-Institute für Gravitationsphysik (Albert-Einstein-Institut) and Leibniz Universität \\ Hannover, Callinstr 38, D-30167 Hannover, Germany \\ 2 Department of Physics, Syracuse University, 201 Physics Building, Syracuse, NY 13244-1130, \\ USA \\ 3 Astrophysics and Space Research Group, School of Physics and Astronomy, \\ University of Birmingham, Birmingham B15 2TT, UK \\ ${ }^{4}$ Institute for Gravitational Research, Department of Physics and Astronomy, \\ University of Glasgow, Glasgow G12 8QQ, UK \\ E-mail: jrsmit02@physics.syr.edu
}

Received 4 September 2007, in final form 30 November 2007

Published 14 January 2008

Online at stacks.iop.org/CQG/25/035003

\begin{abstract}
This paper describes measurements and simulations related to power fluctuations of the laser light in the GEO 600 laser-interferometric gravitational wave detector. Measurements of the relative fluctuations of the light power at three different ports of the main interferometer are presented. In addition, measurements and simulations of the coupling transfer functions from power fluctuations at the input laser to these ports are shown. The transfer function from the input laser to the output port of the interferometer is found to be non-trivial. Despite this, the numerical simulation produces an excellent match to it and gives insight to the mechanisms leading to the complicated shape. Furthermore, the coupling transfer functions of power fluctuations to the main (heterodyne) detector outputs are measured and simulated. These are used to evaluate the level with which laser power fluctuations contribute to the overall noise level of the instrument.
\end{abstract}

PACS numbers: $04.80 . \mathrm{Nn}, 95.55 . \mathrm{Ym}$

(Some figures in this article are in colour only in the electronic version)

\section{Introduction}

A worldwide network of laser-interferometric gravitational wave (GW) detectors is currently in operation [1-4], and a second generation of these with significantly higher sensitivity is planned for the next decade. These instruments are all based on a Michelson interferometer, 
but they employ a variety of other optical techniques, such as power- and signal-recycling and Fabry-Perot cavities, to achieve better sensitivity to gravitational wave strain than a conventional Michelson interferometer.

A thorough understanding of the noise sources that contribute to limiting the sensitivity of today's interferometers is crucial for both improving the existing detectors and designing and commissioning the next generation of detectors to be sensitive enough to routinely detect gravitational waves. Much of the design for future detectors is based on numerical simulations. Therefore, it is desirable to demonstrate the accuracy of these simulations by reproducing the effects measured in the real (and imperfect) systems of current detectors. This is also a valuable way to learn about the mechanisms coupling noise sources to the detector outputs.

One important source of noise for laser interferometers is power noise ${ }^{5}$, i.e., fluctuations of the power of the laser light. Power noise can be caused by mechanisms related to the laser system, fluctuations of the alignment of the interferometer optics, scattered light and other technical noise sources. This noise source led to the development of the heterodyne readout technique, currently used by all interferometric GW detectors to reduce the level with which audio-frequency power noise on the carrier light couples to the detector output. To further reduce the contribution of power noise to the detector noise budgets, all current detectors employ extensive laser power stabilization systems, see [5-8]. Despite these measures, power noise has been shown to contribute to the noise budgets of the gravitational wave detectors, see e.g. [9]. This paper will focus on the level and coupling of laser power noise in GEO 600.

For the measurements presented in this paper, power noise was probed by shining a beam of light from the interferometer onto a photodiode and computing the amplitude spectral density (ASD) of the light power fluctuations. From this, one can calculate the relative power noise (RPN),

$$
\operatorname{RPN}(f)=\frac{\delta P(f)}{\langle P\rangle},
$$

where $\delta P(f)$ is the ASD of power fluctuations on the photodiode at a given Fourier frequency, and $\langle P\rangle$ is the time-averaged light power incident on the photodiode. It is common to normalize in this way because the power noise of a given light field often scales with its dc power.

Measurements of power noise of the light at three ports of the GEO 600 interferometer are presented in section 2. Section 3 shows measured power noise transfer functions from the input laser through the optical system to these three ports and compares them with numerical simulations. In section 4 , these transfer functions and the power noise measurements from section 2 are used to evaluate the level with which the stabilized power input to the interferometer couples to the power in a single arm of the interferometer and to the output port. Coupling transfer functions from power noise to the heterodyne readout detector output signals are presented in section 5 , and these are used to project the level with which power noise contributes to the overall noise level of GEO 600 in section 6.

\section{Measurements of stabilized power noise}

A simplified optical layout of GEO 600 is shown in figure 1. The Michelson interferometer is formed by the beamsplitter and the two $1200 \mathrm{~m}$ long arms comprised of one folding mirror and one end mirror each. Two additional mirrors, the power-recycling mirror and signalrecycling mirror, form a coupled cavity with the Michelson interferometer, making GEO 600 a dual-recycled Michelson interferometer (DRMI). Phase modulation at $F_{\mathrm{MI}} \approx 15 \mathrm{MHz}$ and

5 This is often referred to as intensity noise, but is nearly always measured and presented in units of power, without note of the area of the photodiode with which this power is measured. Therefore, power noise seems more appropriate. 


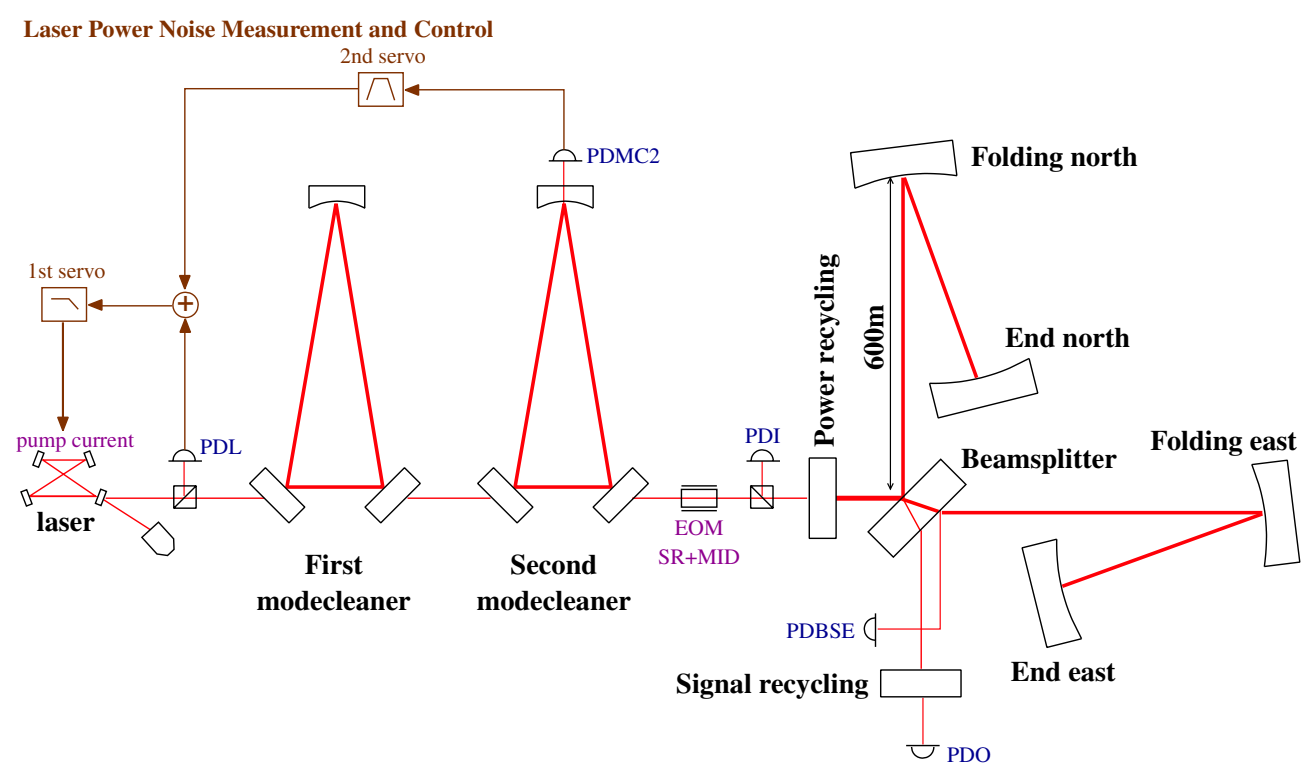

Figure 1. Optical layout of GEO 600 showing the main optics and beams as well as the photodiodes used to measure power noise, and the two power noise stabilization control loops.

$F_{\mathrm{SR}} \approx 9 \mathrm{MHz}$ is imposed on the laser light at the output of the second modecleaner using an electro-optic modulator. A heterodyne scheme then derives error signals from the beat of these control sidebands with the carrier at different ports of the interferometer for the length control of the differential length of the Michelson interferometer, and the length of the signal-recycling cavity, respectively. The frequency of the input laser light is stabilized to the length of the power-recycling cavity. For more information about GEO 600 see [10].

The laser is a $10 \mathrm{~W} \mathrm{Nd:YAG} \mathrm{injection} \mathrm{locked} \mathrm{master-slave} \mathrm{system,} \mathrm{described} \mathrm{in} \mathrm{[5].}$ Noise on the power of the laser output is monitored using an auxiliary beam measured at PDL and actively stabilized by the first power noise control loop, using the slave laser pump current for actuation.

This stabilized light power is then passed through two triangular ring-cavity modecleaners in series before reaching the power-recycling mirror. These cavities are designed to suppress fluctuations in the laser beam geometry [11]. Many effects, such as alignment fluctuations of the input beam relative to the modecleaner eigenmodes, are converted by the modecleaners into additional power noise of the light at their outputs. Because of this, the relative power noise at the output of the second modecleaner is significantly higher than that at the input to the first modecleaner. For this reason, a second control loop is used to stabilize the power of a beam transmitted by the second modecleaner, measured at PDMC2. The control signal of this loop is added to the error signal of the first control loop.

The pre-stabilized power noise injected into the Michelson interferometer is further filtered by the power-recycling cavity and to some extent enhanced by the signal-recycling cavity, before arriving on the output photodiode where power fluctuations can add noise to the main detector output ${ }^{6}$. All of the measurements in this paper were performed during maintenance

6 Power noise can also add noise to the detector output by moving the optics at frequencies in the detection band via radiation pressure. These effects are small for the current configuration of GEO 600 and are thus neglected in this paper. 


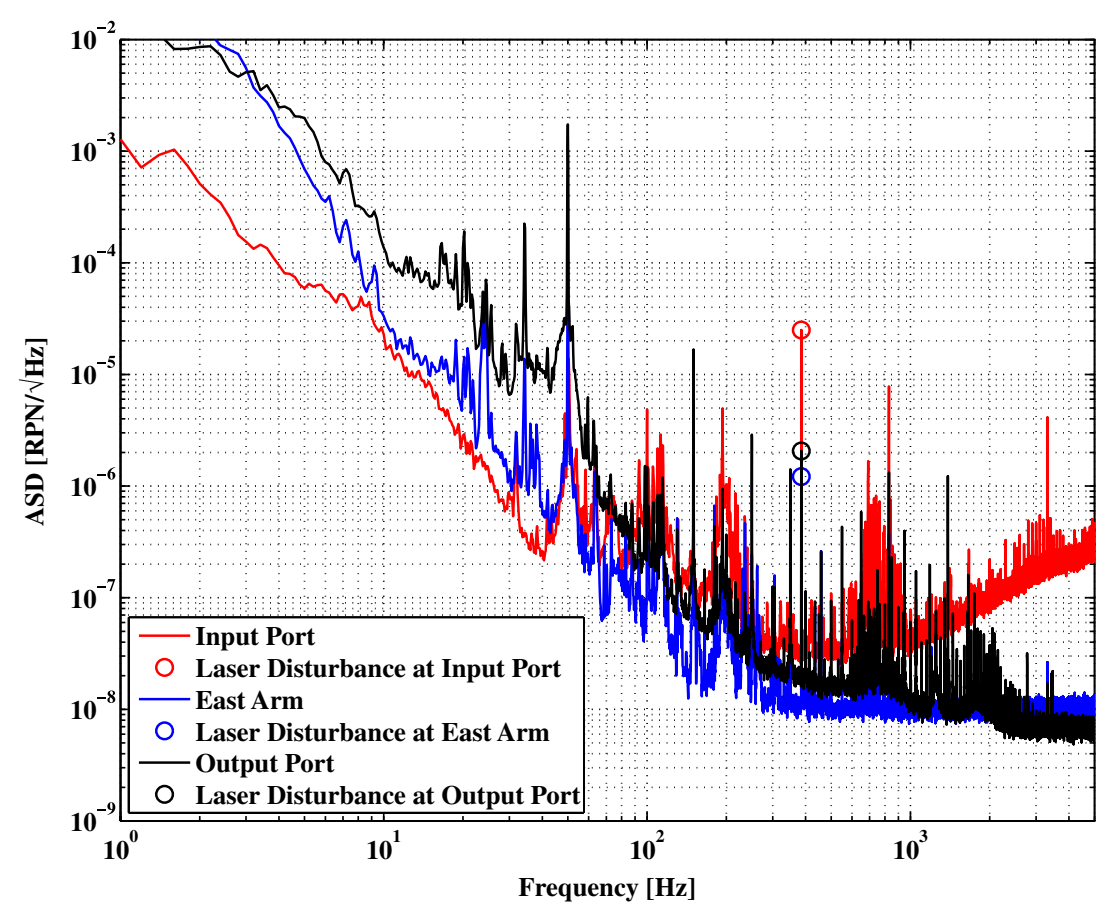

Figure 2. Measured spectra of relative power noise at the input port, east arm and output port of the dual-recycled Michelson interferometer. The circles mark the level with which a periodic disturbance added to the laser power couples to each port.

periods of the S5 science data taking run during which GEO 600 was operated with a signalrecycling cavity detuning frequency of roughly $550 \mathrm{~Hz}$.

Figure 1 also shows the photodiodes used to make measurements of power noise at ports of the DRMI. The photodiode PDI senses the beam reflected by the power-recycling mirror and picked off by a Faraday isolator between the second modecleaner and the interferometer. This is the first photodiode after the second modecleaner that is sensitive to power noise over a broad frequency band. It is therefore useful for measuring power noise on the light injected into the DRMI. However, when the DRMI is locked, PDI senses not only the stabilized incoming light reflected by the power-recycling mirror, but also light that has circulated in the power-recycling cavity and is back transmitted by the power-recycling mirror ${ }^{7}$, as well as so-called junk light from spatial modes rejected by the power-recycling cavity. For these reasons, power noise measurements of the input port light in this paper were made using PDI with the DRMI unlocked and misaligned so that PDI sensed only the input light.

The light from the east arm of the Michelson interferometer that is reflected by the antireflective coating of the main beamsplitter is sensed by PDBSE. The light exiting through the signal-recycling mirror to the output port is sensed by PDO. Measurements from PDBSE and PDO in this paper were made with the DRMI locked in its nominal operating conditions.

Figure 2 shows the relative power noise measured at each of these photodiodes. An RPN of $\approx 2 \times 10^{-8} \mathrm{~Hz}^{-1 / 2}$ is achieved above $300 \mathrm{~Hz}$ at the output port. The circles on the plot mark the amplitude with which a monochromatic power disturbance added to the laser via the pump

7 These light fields have roughly the same dc level on PDI (simulations yield $2.16 \mathrm{~W}$ reflected input power and $1.75 \mathrm{~W}$ back-transmitted power). 
current actuator couples to each of these points. The trace labeled 'input port' corresponds to PDI and gives a measurement of the power noise injected into the interferometer. This can be compared to the power noise at the inputs of the other GW laser interferometers. For example, comparison with that of LIGO [6] shows that the GEO $600 \mathrm{RPN}$ is lower around $1 \mathrm{kHz}$, but is more than two orders of magnitude higher at $10 \mathrm{~Hz}$.

\section{Transfer functions from laser to DRMI ports}

All of the interferometric detectors built thus far employ optical cavities formed by two or more mirrors and used to reduce higher order spatial modes and to resonantly enhance the light power available to interact with gravitational wave strain. On resonance for the fundamental mode, these cavities also act as a single pole filter for power noise with corner frequency

$$
f_{\mathrm{c}}=\frac{\mathrm{FSR}}{2 \mathcal{F}}
$$

where $\mathcal{F}$ is the cavity finesse and the free-spectral range of the cavity is,

$$
\mathrm{FSR}=\frac{c}{l_{\mathrm{c}}},
$$

where $l_{\mathrm{c}}$ is the round-trip optical path length of the cavity. If higher order spatial modes cannot be neglected, if coupled cavities are investigated, or if cavities are detuned from their resonances (as for the signal-recycling cavity of GEO 600), analytical calculations become more difficult. For these cases, it is helpful to calculate the cavity transfer functions using a numerical simulation tool. For the following, the interferometer simulation package FINESSE [15] was used together with an input file ${ }^{8}$ containing the best measured or estimated optical parameters for GEO 600 during S5.

Figure 3 shows the transfer functions from power noise of the laser output to power noise of the light at three later points, namely the second modecleaner, the east arm and the output port. A band-passed random noise ${ }^{9}$ was added to the laser output power via the pump current actuator at a level sufficient to dominate the nominal signal on all of the photodiodes involved in the measurements over the range $\simeq 7-4000 \mathrm{~Hz}$, and the linear response was measured. Nonlinear effects were not included in the measurements.

The transfer function from the laser output to the second modecleaner consists of two single poles, the cavity poles of each modecleaner. This, and additional measurements made to higher frequencies, found the modecleaner poles to be $f_{\mathrm{c}} \simeq 9.0$ and $7.4 \mathrm{kHz}$, for the first and second modecleaner, respectively. The modecleaners are both of length $l_{\mathrm{c}} \simeq 8 \mathrm{~m}$ and thus they have finesse $\mathcal{F} \simeq 2100$ and 2500, respectively, following equation (2). Comparison with $[16,17]$ shows that the finesse of the modecleaners has not changed significantly since 2002, when they were measured to have individual finesses of 1900 and 2700 . No direct measurements of the losses of the individual cavity mirrors have been done since their initial installation. The manufacturer specified scatter loss for these surfaces was between 23 and $37 \mathrm{ppm}$ for each of the mirrors installed [17]. The FINESSE numerical simulation, also shown in figure 3 , reproduces the measured cavity pole frequencies when the modecleaners have a loss of 160 and $130 \mathrm{ppm}$ per high-reflectivity optical surface, respectively. The origin of this excess loss within the modecleaners is not currently understood.

8 This file is available from the authors upon request.

9 The random noise was provided by an Agilent 35670A spectrum analyzer with a first-order bandpass. It was further band-passed by an SRS 560 preamplifier. The gain and filter corner frequencies were chosen on a signal-by-signal basis to give a dominating disturbance over as much of the measurement band as possible, while not producing a significant disturbance below about $5 \mathrm{~Hz}$ in order to not increase the root-mean-squared level of the power noise or the differential arm length fluctuations of the Michelson interferometer. 

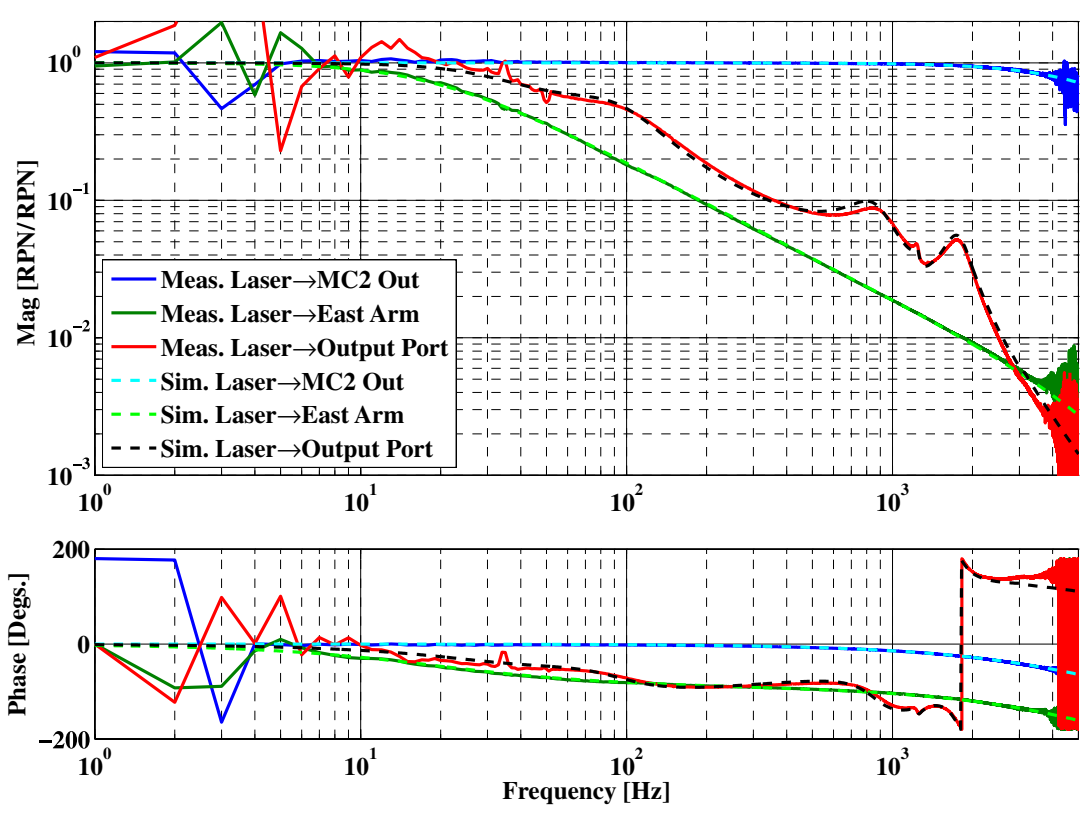

Figure 3. Measured transfer functions from power noise of the laser output to power noise of the light transmitted by the second modecleaner, light in the east arm of the Michelson interferometer and light at the output port. Coherence between the laser output and the measurement channels was achieved over the range $7 \mathrm{~Hz}-4 \mathrm{kHz}$. Also shown are the simulated transfer functions produced with FINESSE.

The transfer function from laser output to the power in the east arm consists of the two modecleaner cavity poles and an additional pole at $\simeq 18.5 \mathrm{~Hz}$ from the power-recycling cavity, which is formed by the power-recycling mirror with $900 \mathrm{ppm}$ transmission and the Michelson interferometer. Again, direct measurements of the individual losses of the optics have not been made. A FINESSE simulation with $130 \mathrm{ppm}$ loss for each high-reflectivity surface and for both surfaces of the beamsplitter matches the measured cavity pole frequency and power buildup factor ( $\simeq 800-900)$. Recent measurements indicate that a loss of roughly this level is caused by small angle scattering from the mirror surfaces.

The transfer function from the laser output to the output port is also shown in figure 3 . It has a roughly similar slope to that from the laser output to the east arm, $f^{-1}$ from $\simeq 50$ to $3000 \mathrm{~Hz}$, but is much more complicated, containing several resonant features. Figure 4 shows this measured transfer function compared with FINESSE simulations performed with carrier light and different combinations of the sideband light at the modulation frequencies $F_{\mathrm{MI}}$ and $F_{\mathrm{SR}}$. For the simulated carrier transfer function, only carrier light was used and the sidebands at $F_{\mathrm{MI}}$ and $F_{\mathrm{SR}}$ were turned off. Similarly for the sideband transfer functions, only the carrier and one pair of sidebands, either $F_{\mathrm{MI}}$ or $F_{\mathrm{SR}}$, were used. The curve labeled, 'Carrier $+F_{\mathrm{MI}}+F_{\mathrm{SR}}$ ' corresponds to the simulation for the carrier and both pairs of sidebands. This is the nominal situation and it matches the measured transfer function very closely.

The carrier light is dominated at the dark port by the $+F_{\mathrm{MI}}$ sidebands with a ratio 2.75:1 due to dark fringe operation for the carrier and a Schnupp asymmetry ${ }^{10}$ in the arms.

\footnotetext{
${ }^{10}$ This refers to a difference in the length of the arms of the Michelson interferometer, introduced to create a locking condition in which the output port is dark for the carrier while not completely dark for the control sidebands. This technique is attributed to Lise Schnupp, formerly of the Garching group.
} 

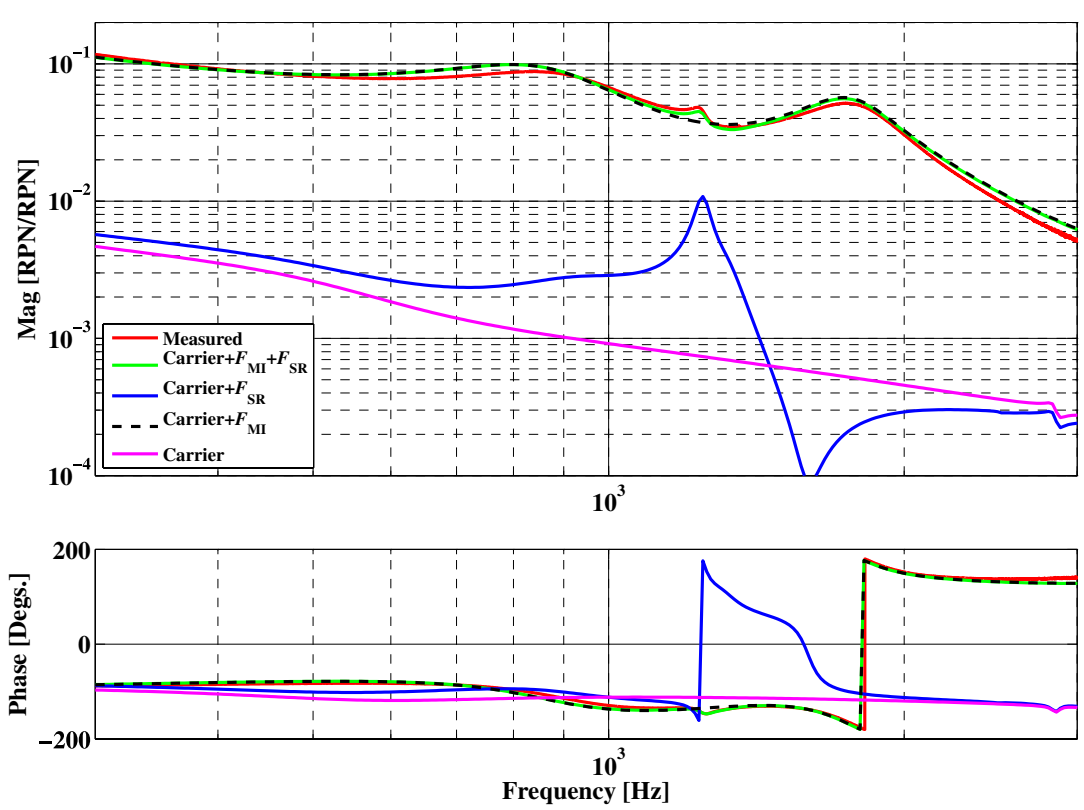

Figure 4. A zoomed-in view of the measured transfer function from power noise of the laser output to power noise at the output port. Also shown are the simulated transfer functions produced with FINESSE with the carrier light and different combinations of the control sidebands.

The simulations indicate that power noise on the Michelson control sidebands gives this transfer function most of its shape. The two broad resonances in the transfer function around 800 and $1500 \mathrm{~Hz}$ are due to the resonance of the Michelson sidebands in the signal-recycling cavity. The simulation also reproduces the small bump in the magnitude of the measured transfer function around $1.2 \mathrm{kHz}$ and shows it to be related to power noise sidebands around the signal-recycling control sidebands, which are resonant in the power-recycling cavity at that frequency.

\section{Stabilized input power noise contribution to DRMI ports}

To motivate decisions about future power noise stabilization strategies, it is useful to know the level with which the stabilized input power noise contributes to the power noise within the interferometer and at the output port. To determine this, the RPN at the interferometer input port (PDI) presented in section 2 is multiplied by the magnitude of the simulated transfer functions from the input port to the east arm and output port (equivalent to those presented in section 3, but without the modecleaner cavity poles). The results represent the RPN in the east arm and output port contributed by the stabilized light at the input port. These can be compared with the RPN measured at PDBSE and PDO to see how much of the power noise measured there comes from the input to the interferometer.

It is also worth noting that GEO 600 may use a dc readout scheme to enhance its sensitivity in the near future. With dc readout, the audio-frequency output of PDO will be the main output signal. Power noise at the dark port will couple directly to this signal, and it will be beneficial to understand the coupling of power noise to the dark port. Although the transfer functions will differ for the two readout schemes, the principles are the same.

Figure 5 shows the RPN measured for the east arm of the Michelson at PDBSE compared to that at PDI multiplied by the magnitude of the simulated transfer function between these 


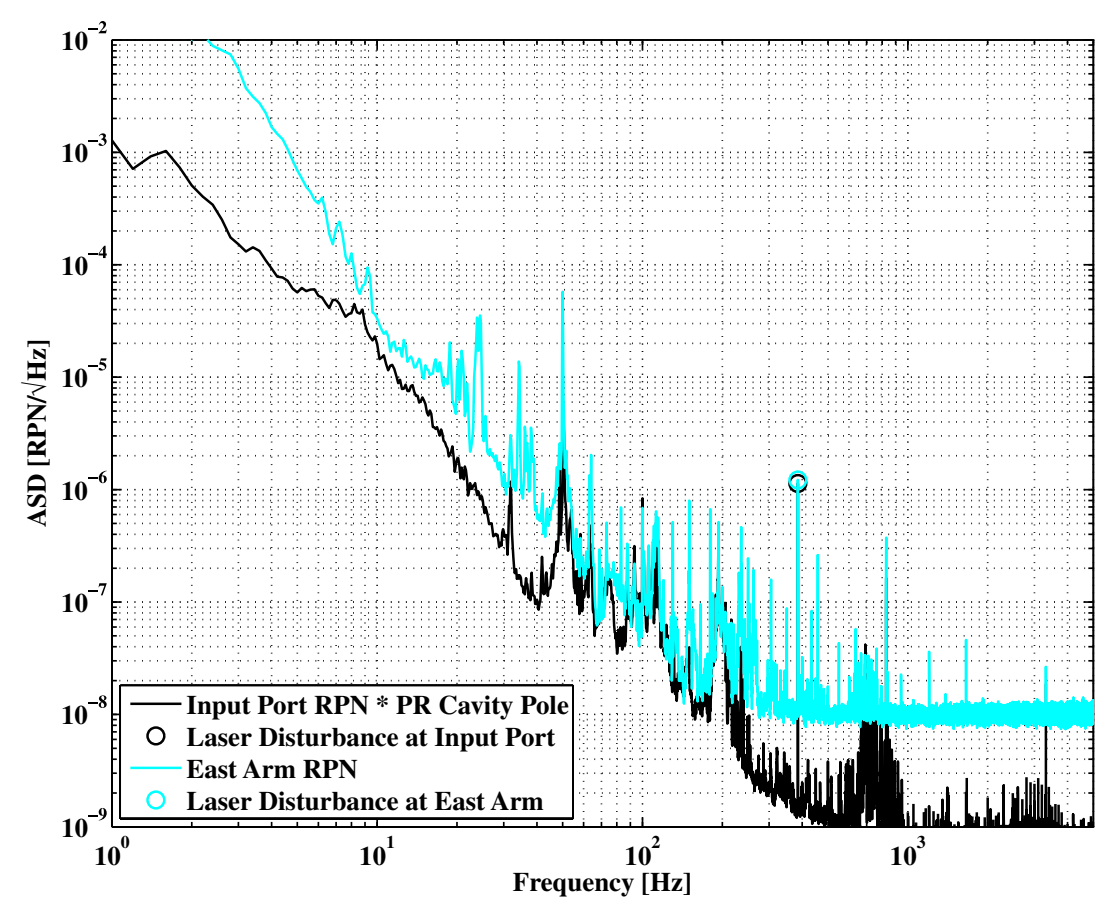

Figure 5. Contribution of power noise at the DRMI input port to that in the east arm. The power noise at the input port is multiplied by the magnitude of the transfer function from input port to the east arm calculated by FINESSE.

ports, i.e., the PR cavity pole. The monochromatic laser power calibration lines (circles) now have the same amplitude, as expected (since in one case the laser disturbance is filtered by the physical system and in the other it is filtered by the simulated transfer function of the same). From this plot one can say that the power noise in the east arm is limited by the stabilized input laser power noise at least over the range $\approx 50-200 \mathrm{~Hz}$. The east arm measurement is limited by shot noise above $200 \mathrm{~Hz}$, thus constituting an upper limit on RPN. Additional measurements show that the excess power noise seen below $50 \mathrm{~Hz}$ is due to noise coupled from the longitudinal and angular control loops of the DRMI.

Figure 6 shows the power noise measured at PDI propagated to the output port by the transfer function calculated by FINESSE. This shows that the stabilized input power noise is limiting the power noise at the output port at noise bumps around 100 and $200 \mathrm{~Hz}$. Above $2 \mathrm{kHz}$, the output port measurement is limited by shot noise. At other frequencies, there is a significantly higher power noise at the output port than can be explained by the power noise of the stabilized input light.

\section{Transfer functions from power noise to the detector outputs}

During S5, the GEO 600 detector used a heterodyne readout scheme and detuned signal recycling. In this configuration, the gravitational wave signal is split between two quadrature demodulations of the signal at PDO, referred to as P (in-phase) and Q (quadrature). These signals are calibrated to equivalent gravitational wave strain and optimally combined to form a single detector output signal [12] used for GW analysis. 


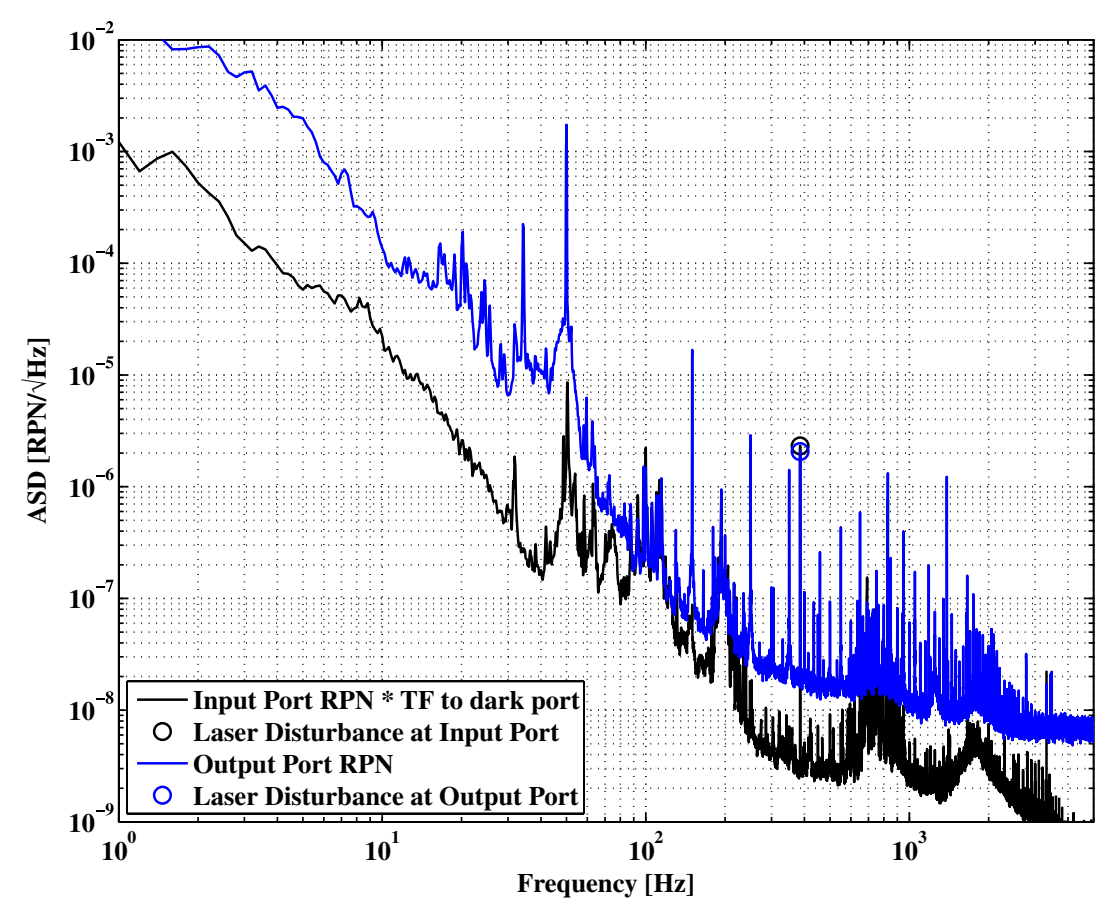

Figure 6. Contribution of power noise at the DRMI input port to that at the output port. The power noise at the input port is multiplied by the magnitude of the transfer function from input port to output port calculated by FINESSE.

Figure 7 shows the transfer functions measured from the power noise at the input port to $\mathrm{P}$ and Q. These injection/response measurements were made with the same random noise laser power disturbance described in section 3. The transfer functions simulated using FINESSE are also shown. The demodulation phases for $\mathrm{P}$ and $\mathrm{Q}$ used in the simulation were set by matching the simulated optical transfer function from differential motions of the end mirrors to a fit of the measured optical transfer function provided by the GEO 600 on-line calibration process $[12,13]$. The measured transfer functions are very complicated, exhibiting several resonance and notch features.

The FINESSE simulations contain most of the features seen in the measurements, although they do not agree as neatly as the sets of power noise to power noise transfer functions shown in figure 3. The behavior of the phase in the simulation around $400 \mathrm{~Hz}$, which goes in the opposite direction as the measured phase, is dependent on the properties of a coupling crossover that causes the notch structure. The phase can be made to fit the data there, with little effect on the rest of the transfer function, by e.g., arbitrarily lengthening the distance between the beamsplitter and the signal-recycling mirror from the measured value of 1.109 to $1.116 \mathrm{~m}$. The notable discrepancies in phase at low frequencies are not yet understood.

\section{Noise projection of power noise to the detector outputs}

Figure 8 shows $\mathrm{P}, \mathrm{Q}$ and noise projections made by multiplying the amplitude spectral density of the power noise at PDI shown in figure 2 by the magnitudes of the measured transfer functions shown in figure 7, as described in [14]. These show that the stabilized input laser 

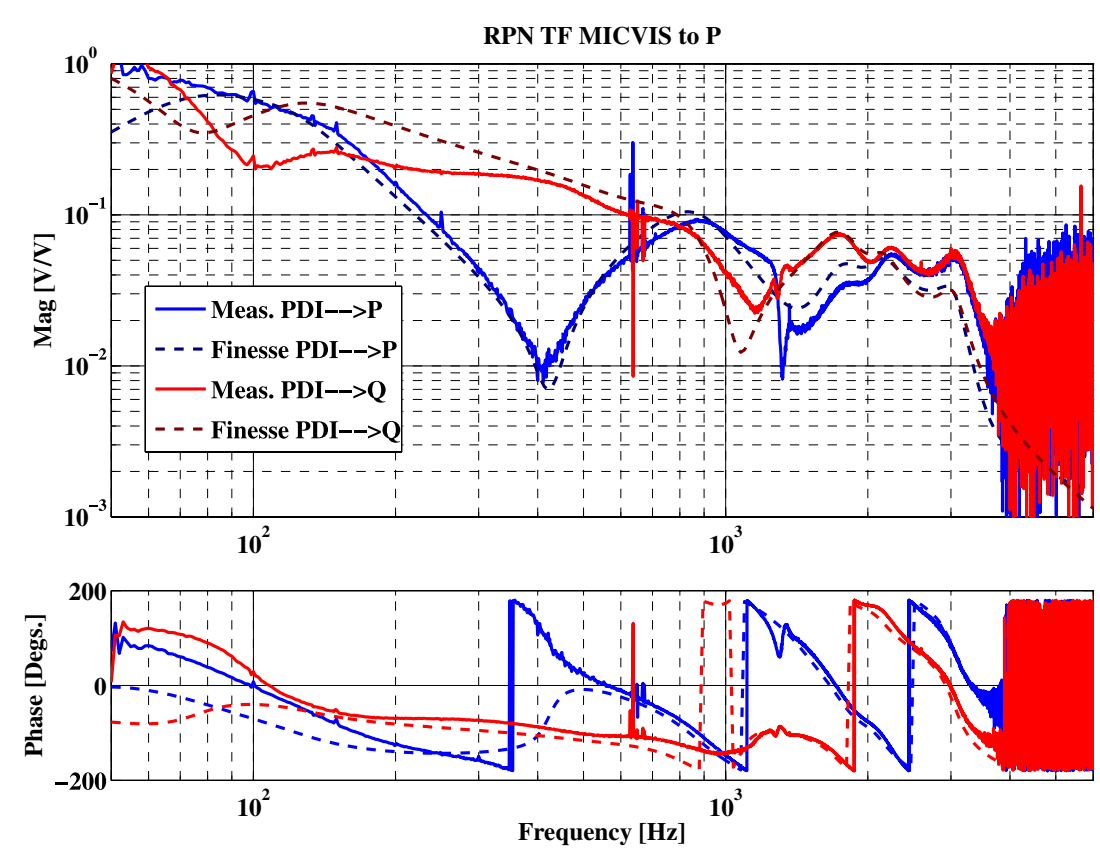

Figure 7. Measured transfer functions from the stabilized laser power input to the DRMI, to the main detector output signals $\mathrm{P}$ and $\mathrm{Q}$. Also shown are the simulated transfer functions produced with FINESSE.

power noise is close to limiting the current sensitivity of GEO 600 around 100-200 Hz, but does not contribute significantly at other frequencies.

Shot noise of the light at the output port sets the theoretical limit to sensitivity for the current configuration of GEO 600 above about $100 \mathrm{~Hz}$. Thus, it is sensible to compare the noise projections of power noise to the level of shot noise in P and Q. An estimate for the level with which shot noise contributes to both $\mathrm{P}$ and $\mathrm{Q}$ is shown in figure 8 . The contributions of power noise at the input port to $\mathrm{P}$ and $\mathrm{Q}$ are well below the shot noise level above $200 \mathrm{~Hz}$, except for at a group of narrow spectral features between about 650 and $850 \mathrm{~Hz}$. However, it should be noted that power noise could become more of a problem for higher laser powers, since the shot noise signal-to-noise ratio (SNR) is expected to increase with the square-root of laser power, while the power noise SNR is expected to be independent of laser power.

\section{Summary}

New measurements of the relative power noise at several ports of GEO 600 were presented in this paper. The active stabilization of the light input to the DRMI, together with the passive filtering of power noise provided by primarily the power-recycling cavity, results in an RPN of $\approx 2 \times 10^{-8} \mathrm{~Hz}^{-1 / 2}$ at the dark port above $300 \mathrm{~Hz}$.

The transfer functions coupling power noise from the laser to three ports of the interferometer were measured, and accurately simulated using FINESSE. These simulations suggest that the optics comprising the two modecleaners and the DRMI have optical losses of 130-160 ppm per highly-reflective surface, on average. The transfer function from the laser to the output port was shown to be complicated. Decomposing it into contributions from the various light fields using FINESSE was helpful in determining that the complication originates 


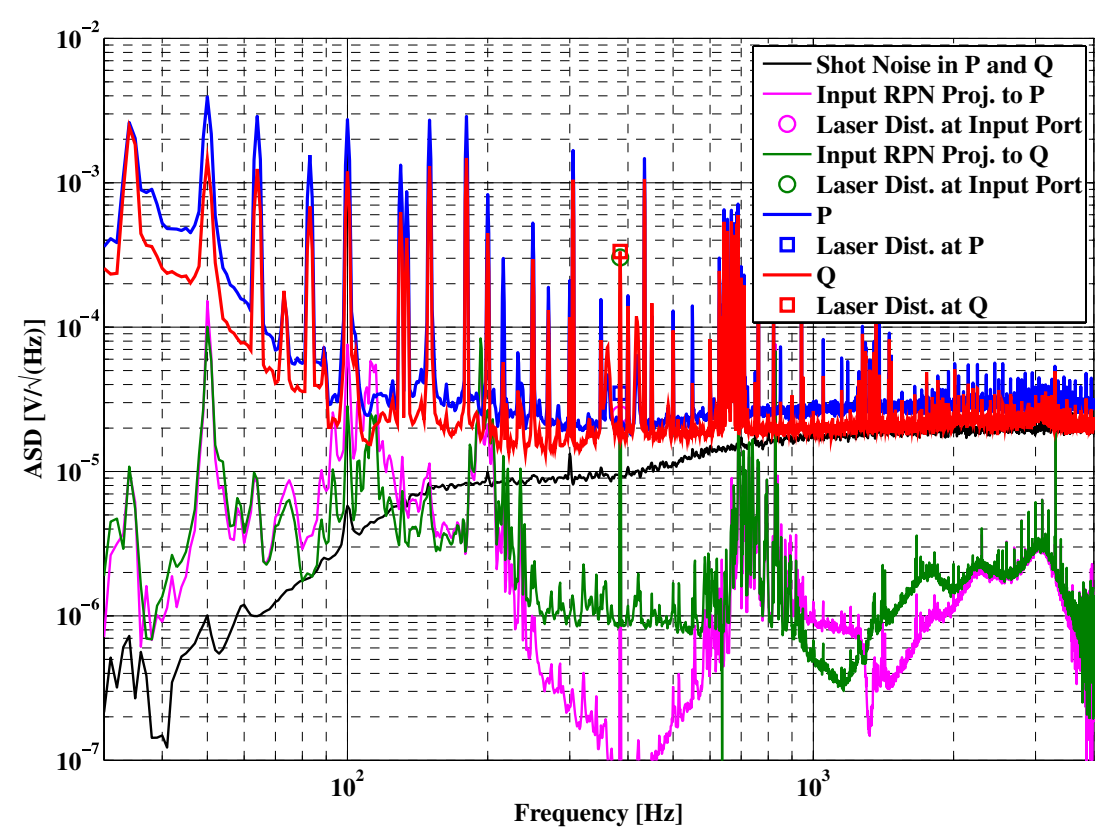

Figure 8. Noise projection from the stabilized laser power noise at the input port of the DRMI to the main detector output signals $\mathrm{P}$ and $\mathrm{Q}$. The expected level of shot noise in $\mathrm{P}$ and $\mathrm{Q}$ for nominal conditions during S5 is also shown for reference. The agreement of the peak heights of the laser disturbances in $\mathrm{P}$ and $\mathrm{Q}$ and the projected noise for each gives an indication of the accuracy of the noise projections.

primarily from the coupling of power noise on the RF control sidebands for the Michelson interferometer and signal-recycling cavities.

These transfer functions were also used to determine the levels with which power noise at the input port of the DRMI couples to the east arm and output port. The input power noise was found to contribute to power noise in the east arm over the range 50-200 Hz and at the output part around 100 and $200 \mathrm{~Hz}$. The power stability at these frequencies could be improved with a further stabilization of the input power. At other frequencies, a significant amount of power noise is added within the DRMI, in part by control signals.

The transfer functions coupling power noise to the main detector outputs were also measured and found to be very complicated. Numerical simulations produced close, but not perfect matches to the measurements. Using the measured transfer functions, the level of power noise was compared to the detector output noise and found to not contribute significantly to the current noise budget, except at noise bumps around 100 and $200 \mathrm{~Hz}$.

This work indicates that the numerical simulation program FINESSE can accurately reproduce transfer functions of a complicated and imperfect optical system. This gives a great deal of confidence in the accuracy of simulations for current and future gravitational wave detectors.

\section{Acknowledgments}

The authors are grateful for support from PPARC and the University of Glasgow in the UK, and the BMBF and the state of Lower Saxony in Germany. We would like to thank the members of the ILIAS-GW N5-WG1 group for useful discussions concerning power noise, simulations 
and noise projections. This document has been assigned LIGO Laboratory document number LIGO-P070092-00-Z.

\section{References}

[1] Willke B et al 2007 GEO600: status and plans Class. Quantum Grav. 24 S389-97

[2] Waldman S J et al 2006 Status of LIGO at the start of the fifth science run Class. Quantum Grav. 23 S653-60

[3] Acernese F et al 2006 The Virgo status Class. Quantum Grav. 23 S635-42

[4] Ando M et al 2002 Current status of TAMA Class. Quantum Grav. 19 S1409-19

[5] Zawischa I 2002 The GEO600 laser system Class. Quantum Grav. 19 1775-81

[6] Nocera F 2004 LIGO laser intensity noise suppression Class. Quantum Grav. 21 S481-5

[7] Nagano S et al 2003 Development of a multistage laser frequency stabilization for an interferometric gravitational wave detector Rev. Sci. Instrum. 74 4176-83

[8] Bondu F et al 2002 The VIRGO injection system Class. Quantum Grav. 19 1829-33

[9] Adhikari A 2004 Sensitivity and noise analysis of $4 \mathrm{~km}$ laser interferometric gravitational wave antennae PhD Thesis MIT

[10] Willke B et al 2004 Status of GEO 600 Class. Quantum Grav. 21 S417-23

[11] Rüdiger A et al 1981 Opt. Acta 28 5, 641-58

[12] Hewitson M et al 2005 Optimal time-domain combination of the two calibrated output quadratures of GEO600 Class. Quantum Grav. 22 4253-61

[13] Hewitson M et al 2004 Principles of calibrating the dual-recycled GEO600 Rev. Sci. Instrum. 75 4702-9

[14] Smith J R et al 2006 Linear projection of technical noise for interferometric gravitational wave detectors Class. Quantum Grav. 23 527-37

[15] Freise A et al 2004 Frequency-domain interferometer simulation with higher-order spatial modes Class. Quantum Grav. 21 S1067-74 (FINESSE is available at http://www.rzg.mpg.de/adf/)

[16] Gossler S et al 2002 The modecleaner system and suspension aspects of GEO600 Class. Quantum Grav. $191835-42$

[17] Freise A 2003 The next generation of interferometry: multi-frequency optical modelling, control concepts and implementation PhD Thesis University of Hannover 